\title{
Insulin-Like Growth Factor-1 and Insulin-Like Growth Factor-Binding Protein-3 Concentrations in Children With Obstructive Sleep Apnea-Hypopnea Syndrome
}

\author{
Jin Zhu MD, Yu Fang MD, Hai-Fei Wang MD MSc, Xin Chen MD MSc, \\ Dao-Jun Yu MD PhD, and Yi Shen MD
}

\begin{abstract}
BACKGROUND: The aim of this study was to assess insulin-like growth factor-1 (IGF-1) and insulin-like growth factor-binding protein-3 (IGFBP-3) concentrations in children with obstructive sleep apnea-hypopnea syndrome (OSAHS) and to compare the results according to disease severity and duration. METHODS: Fifty-one children with OSAHS underwent polysomnography and were classified as having mild, moderate, or severe disease. All children underwent bilateral tonsillectomy/adenoidectomy or adenoidectomy. IGF-1 and IGFBP-3 concentrations were assessed before surgery and 3 and 6 months after surgery. RESULTS: Before surgery, IGF-1 and IGFBP-3 concentrations were significantly higher $(P<.05)$ in the $<5$-y group $(125.20 \pm 42.38 \mu \mathrm{g} / \mathrm{L}$ and $2.00 \pm 0.54 \mathrm{mg} / \mathrm{L})$ compared with the $\geq 5$-y group $(61.92 \pm 19.07 \mu \mathrm{g} / \mathrm{L}$ and $1.20 \pm 0.37 \mathrm{mg} / \mathrm{L})$. After surgery, concentrations significantly increased $(P<.05)$ in both groups, but remained lower in the $\geq 5-y$ group. Before surgery, there were no significant differences in IGF-1 (mild: 93.82 $\pm 37.52 \mathrm{mg} / \mathrm{L}$; moderate: $109.06 \pm 58.42 \mathrm{mg} / \mathrm{L}$; severe: $73.88 \pm 28.51 \mathrm{mg} / \mathrm{L}$ ) and IGFBP-3 (mild: $1.76 \pm 0.57 \mathrm{mg} / \mathrm{L}$; moderate: $1.67 \pm 0.72 \mathrm{mg} / \mathrm{L}$; severe: $1.33 \pm 0.45 \mathrm{mg} / \mathrm{L}$ ) concentrations by severity. After surgery, concentrations significantly increased $(P<.05)$ in both groups, but did not differ by severity. After adjusting for time after surgery, disease duration, apneahypopnea index, time spent at $\mathrm{S}_{\mathrm{pO}_{2}}<90 \%$, and body mass index $\mathrm{Z}$ score, IGF-1 and IGFBP-3 concentrations significantly decreased with every year of age. CONCLUSIONS: These results suggest that disease duration, but not severity, affects the concentrations of 2 important mediators of growth/development (IGF-1 and IGFBP-3) in children with OSAHS before and after surgery. Key words: children; duration; development; disease; growth; obstructive sleep apnea-hypopnea syndrome; pediatric; severity. [Respir Care 2015;60(4):593-602. (C) 2015 Daedalus Enterprises]
\end{abstract}

\section{Introduction}

Estimates suggest that $1-3 \%$ of children experience obstructive sleep apnea-hypopnea syndrome (OSAHS), ${ }^{1,2}$ which

\footnotetext{
Drs Zhu, Fang, and Chen are affiliated with the Department of Otorhinolaryngology Head and Neck Surgery, and Dr Yu is affiliated with the Gene Laboratory, Hangzhou First People's Hospital (affiliated with Nanjing Medical University), Zhejiang, China. Dr Wang is affiliated with Zhongda Hospital (affiliated with Southeast University), Nanjing, China. Dr Shen is affiliated with the School of Public Health, Zhejiang University, Zhejiang, China.

Supplementary material related to this paper is available at http:// www.rcjournal.com.
}

is typically caused by adenoidal and/or tonsillar hypertrophy. ${ }^{3,4}$ In addition to the overt clinical manifestations (ie,

\footnotetext{
This study was supported by grant 20100633B03 from the Science and Technology Development Project of Hangzhou and grant 2012C33036 from the Science and Technology Plan Program of Zhejiang Province.

The authors have disclosed no conflicts of interest.

Correspondence: Jin Zhu MD, Department of Otorhinolaryngology Head and Neck Surgery, Hangzhou First People's Hospital (affiliated with Nanjing Medical University), 261 Huansha Road, Hangzhou 310006, China. E-mail: zhujin2698@163.com.
}

DOI: $10.4187 /$ respcare. 03516 
snoring and mouth breathing), children with OSAHS may experience poor appetite, neurocognitive/behavioral issues, and impaired growth/failure to thrive. ${ }^{5,6}$ As childhood is a critical time of growth and development, appropriate and timely treatment is of paramount importance.

Surgery is the standard treatment for OSAHS caused by adenoidal and/or tonsillar hypertrophy in children. Of note, findings from a number of studies indicate that children with OSAHS who undergo adenoidectomy and/or tonsillectomy experience various improvements, including rate of growth. ${ }^{5,7}$ The decreased growth before surgery and improved growth after surgery would appear to be mediated, at least in part, by alterations in growth hormone and associated factors, namely insulin-like growth factor-1 (IGF-1) and its major carrier/binding protein, insulin-like growth factor-binding protein-3 (IGFBP-3), both of which are commonly used measures of growth and development and reflect growth hormone secretion. ${ }^{8-11}$ Indeed, it has been suggested that the normal pulsatile secretion of growth hormone from somatotrophs may be adversely affected by the abnormal sleep rhythms in children with OSAHS. ${ }^{12,13}$ Strong support for the involvement of IGF-1 and IGFBP-3 in contributing to growth deficits in children with OSAHS comes from the findings of a meta-analysis published in $2009,{ }^{7}$ which demonstrated that standardized height and weight and IGF-1 and IGFBP-3 concentrations significantly increased following adenotonsillectomy.

Although there is reasonably strong evidence that perturbations in IGF-1 and IGFBP-3 play a role in mediating the growth deficits in children with OSAHS, there is little information available on how the severity of OSAHS or the duration of disease affects these parameters. We believe that determining whether IGF-1 and IGFBP-3 concentrations in children with OSAHS are affected by these variables is important not only to increase our understanding of the condition but also to potentially aid in clinical decision-making. Hence, the aim of this study was to examine IGF-1 and IGFBP-3 concentrations in children with OSAHS before and after interventional surgery and to make comparisons on the basis of disease severity (as determined by polysomnography) and disease duration. We also assessed changes in apnea-hypopnea index (AHI) and lowest oxygen saturation.

\section{Methods}

\section{Subjects}

This was a retrospective analysis of prospective data obtained from the records of children with OSAHS who were treated in the Department of Otolaryngology of the Hangzhou First People's Hospital (affiliated with Nanjing Medical University) from February 2010 to October 2011.

\section{QUICK LOOK}

\section{Current knowledge}

Surgery is the standard treatment for obstructive sleep apnea caused by adenoidal and/or tonsillar hypertrophy in children. In children with obstructive sleep apneahypopnea syndrome who undergo adenoidectomy and/or tonsillectomy, the rate of growth has been shown to accelerate.

\section{What this paper contributes to our knowledge}

Obstructive sleep apnea caused by adenoidal and/or tonsillar hypertrophy reduces the concentrations of 2 key mediators of growth and development in children. Adenoidectomy and/or tonsillectomy significantly increased the concentrations of growth mediators regardless of disease severity. Prompt treatment may help alleviate the potential impact on growth and development.

The study was approved by the independent ethics committee of the Hangzhou First People's Hospital. Informed consent was obtained for all children from their families.

Most of the children in this study were brought for treatment because of parent-reported snoring or sleep arousal. Others were brought because their parents had scientific knowledge of OSAHS or had heard that other children recovered after treatment, and the parents suspected that their child had the disease. Some subjects were referred by pediatricians, psychologists, or dentists due to hyperactivity, inattention, restlessness, or abnormalities of the palate. Through detailed interviews, especially in confirming the medical records showing the first diagnosis and strict screening, recall bias was minimized.

All children suspected to have OSAHS in our clinics underwent polysomnography monitoring to determine whether they had OSAHS and, if so, what type (central, peripheral, or mixed). The combined results of diagnostic imaging were used to determine whether surgery was warranted in each case. However, polysomnography is not generally a routine test that is conducted before preoperative treatment and assessment.

Because the included subjects still needed to undergo some unconventional serological testing in addition to conventional treatment, the subjects and parents were informed of the purpose of the study, specific processes, and procedures. If the parents did not agree to participate in the study group, the children were treated with conventional therapies. If they fully understood and agreed to partici- 


\section{Growth and Development of Children With OSAHS}

pate in the study, informed consent forms were signed by the parents, and the children were included. The families of the subjects were informed that the children could withdraw from the study at any time, without justification, and their treatment would not be affected.

\section{Inclusion Criteria}

Children with OSAHS were considered for inclusion in the study if they underwent polysomnography and met the criteria for childhood OSAHS according to the 2007 guidelines for diagnosis and treatment of OSAHS in children (Otolaryngology Branch, Chinese Medical Association) ${ }^{14}$; had a normal history of birth, feeding, and growth; were 5-12 y old; experienced OSAHS onset at $3 \mathrm{y}$ of age based on the subjects' medical record and the parents' recollection; were prepubertal; and had a stable living environment.

OSAHS onset at $3 \mathrm{y}$ of age represented the majority of cases in our clinic. For the convenience of screening, children with an onset at $3 \mathrm{y}$ were chosen. The exact time of onset in children was calculated to determine the disease duration (child's age - onset age $=$ disease duration). If the time of onset (in specific subjects) was difficult to accurately determine, those individuals were excluded because accuracy of the study results would have been affected.

Seventy subjects were selected from more than $500 \mathrm{pa}-$ tients and followed for $>2 \mathrm{y}$. Fifty-one subjects completed the entire course of the study. The disease course was distinguished as being $\geq 5$ or $<5$ y in duration because: (1) OSAHS is a chronic disease, so the symptoms are not obvious in the short-term; and (2) currently, there are no international standards for the disease course of OSAHS, and from our OSAHS diagnosis and treatment experience and observation (although without clinical evidence), the symptoms are generally obvious in children with a disease course of $>5 \mathrm{y}$, so the $<5$-y and $\geq 5$-y classifications for the study groups were used. Seventytwo healthy children (non-OSAHS or without growth abnormalities) from the same age group as the experimental group (5-12 y of age) who had undergone routine physical examinations were randomly selected to serve as a control group.

\section{Exclusion Criteria}

Children were excluded from the study if they had any congenital anatomic abnormality of the mouth, nose, or pharynx; experienced severe asphyxia or hypoxic-ischemic encephalopathy at birth; chronic diseases such as tuberculosis, viral hepatitis, or nephrotic syndrome; a history of major trauma, cerebral palsy, epilepsy, or repeated up- per respiratory tract infection; a family history of familial short stature, congenital mental retardation and mental illness, or any other disease potentially affecting growth and development (eg, genetic or metabolic diseases); or OSAHS not caused by adenoid hyperplasia and/or tonsillar hypertrophy.

\section{Surgery}

All children underwent bilateral tonsillectomy and adenoidectomy or adenoidectomy alone and recovered uneventfully.

\section{Assessments}

Children were divided into 2 groups according to the duration of OSAHS: $<5 \mathrm{y}$ or $\geq 5 \mathrm{y}$. According to the guidelines for diagnosis and treatment of OSAHS in children, ${ }^{14}$ the diagnosis of OSAHS is based primarily on the AHI, whereas the lowest oxygen saturation is supplementary information. OSAHS was classified as mild $(5<\mathrm{AHI} \leq 10$ times $/ \mathrm{h})$, moderate $(10<\mathrm{AHI} \leq$ 20 times/h), or severe (AHI $>20$ times/h). ${ }^{14}$ Polysomnography was performed using a multi-channel sleep monitoring system (Embletta X100, Embla, Broomfield, Colorado) for $>7 \mathrm{~h}$ before surgery and 3 and 6 months after surgery. The AHI and lowest oxygen saturation were determined during each polysomnography assessment. Body mass index (BMI) measurements were also taken at each time point. Venous blood samples were collected before surgery and 3 and 6 months after surgery.

We enrolled a total of 70 subjects and extracted $3 \mathrm{~mL} /$ patient/time point from blood samples. In addition, 51 subjects exhibited good compliance and completed the 3 blood collection time points according to the experimental design requirements. Serum was obtained by centrifugation, and IGF-1 and IGFBP-3 concentrations were determined by chemiluminescence enzyme-linked immunosorbent assay using a commercially available kit (Siemens Healthcare Diagnostics, Tarrytown, New York). The IGF-1 and IGFBP-3 detection device used was the DPC Immulite 2000 automatic immune analyzer (Siemens Healthcare Diagnostics) and its original reagents. According to the reagent manual supplied by the manufacturer, the normal reference ranges of IGF- 1 and IGFBP-3 are $64-345 \mu \mathrm{g} / \mathrm{L}$ and $1.6-6.5 \mathrm{mg} / \mathrm{L}$, respectively.

\section{Sample Size Estimate}

The study sample size was estimated according to the repeated measurement method: mean correlation coefficient $=0.5$, effect size $=0.57$, repeated measurements $=3$, power $=0.8$, and $\alpha$ level $=.05$. This estimation revealed 


\section{Growth and Development of Children With OSAHS}

Table 1. Summary of Baseline Demographic and Disease Characteristics by Disease Duration in Children With Obstructive Sleep ApneaHypopnea Syndrome

\begin{tabular}{|c|c|c|c|}
\hline \multirow{2}{*}{ Characteristic } & \multicolumn{2}{|c|}{ Disease Duration } & \multirow{2}{*}{$P$} \\
\hline & $<5$ y $(n=25)$ & $\geq 5$ y $(n=26)$ & \\
\hline Age (mean $\pm S D), y$ & $5.88 \pm 0.83$ & $9.42 \pm 1.47$ & $<.001$ \\
\hline Sex, $n(\%)$ & & & .19 \\
\hline Male & $15(60)$ & $20(76.92)$ & \\
\hline Female & $10(40)$ & $6(23.08)$ & \\
\hline Severity, $n(\%)$ & & & .38 \\
\hline Mild & $8(32)$ & $9(34.62)$ & \\
\hline Moderate & $11(44)$ & $7(26.92)$ & \\
\hline Severity & $6(24)$ & $10(38.46)$ & \\
\hline $\mathrm{BMI}($ mean $\pm \mathrm{SD}), \mathrm{kg} / \mathrm{m}^{2}$ & $17.07 \pm 2.98$ & $18.99 \pm 5.33$ & .12 \\
\hline BMI Z score (mean \pm SD) & $0.71 \pm 1.3$ & $0.32 \pm 1.45$ & .33 \\
\hline $\mathrm{AHI}($ mean $\pm \mathrm{SD})$ & $16.92 \pm 11.42$ & $17.98 \pm 9.06$ & .72 \\
\hline Lowest oxygen saturation (mean $\pm \mathrm{SD}$ ) & $0.77 \pm 0.09$ & $0.77 \pm 0.07$ & .94 \\
\hline Lowest oxygen level (mean \pm SD), $\%$ & $77.48 \pm 9.19$ & $77.31 \pm 7.23$ & .94 \\
\hline $\mathrm{S}_{\mathrm{pO}_{2}}($ mean $\pm \mathrm{SD}), \%$ & $83.16 \pm 4.91$ & $83.68 \pm 4.42$ & 69 \\
\hline Time spent at $\mathrm{S}_{\mathrm{pO}_{2}}<90 \%$ (mean $\pm \mathrm{SD}$ ), $\%$ & $76.68 \pm 22.03$ & $78.8 \pm 18.58$ & .71 \\
\hline $\mathrm{IGF}-1$ (mean $\pm \mathrm{SD}), \mu \mathrm{g} / \mathrm{L}$ & $125.2 \pm 42.38$ & $61.92 \pm 19.07$ & $<.001$ \\
\hline Abnormal IGF-1, $n(\%)$ & $0(0)$ & $15(57.69)$ & $<.001$ \\
\hline IGFBP-3 (mean $\pm \mathrm{SD}), \mathrm{mg} / \mathrm{L}$ & $2.00 \pm 0.54$ & $1.20 \pm 0.37$ & $<.001$ \\
\hline Abnormal IGFBP-3, $n(\%)$ & $5(20 \%)$ & $21(80.77 \%)$ & $<.001$ \\
\hline $\begin{array}{l}\text { BMI }=\text { body mass index } \\
\text { AHI }=\text { apnea-hypopnea index } \\
\text { IGF-1 = insulin-like growth factor- } 1 \\
\text { IGFBP-3 = insulin-like growth factor-binding protein-3 }\end{array}$ & & & \\
\hline
\end{tabular}

that a sample size of at least 14 participants was required for each group.

\section{Statistical Analysis}

Continuous variables are presented as mean \pm SD and were compared by the independent sample $t$ test (2 groups) or one-way analysis of variance with the Bonferroni post hoc test ( 3 or more groups). Categorical variables are presented as count (percentage) and were compared between groups by a chi-square test. For repeated measures (IGF-1, IGFBP-3, AHI, lowest oxygen saturation, BMI Z score, weight, height, and $\mathrm{S}_{\mathrm{pO}_{2}}$ factors), linear mixed models were used to investigate the effect of groups (denoted as group effect), timing of assessment (denoted as time effect), and their interaction (denoted as group $\times$ time effect). When significance was detected for the main effect or interaction, Bonferroni correlations were used to control for the type- 1 error rate during post hoc multiple comparisons. Statistical analyses were performed using SAS 9.2 (SAS Institute, Cary, North Carolina). A 2-tailed $P$ value of $<0.05$ was considered to indicate statistical significance.

\section{Results}

A total of 70 children with OSAHS were eligible for inclusion in the study. Of these, 19 were lost to follow-up or had incomplete follow-up; hence, 51 children with OSAHS who had complete medical records ( 35 boys and 16 girls) were included in the analyses.

\section{Demographic and Clinical Characteristics}

Children in the OSAHS group had lower IGF-1 and IGFBP-3 concentrations (month 0/baseline), and the concentration of IGF-1 and IGFBP-3 increased gradually after 3 and 6 months after surgery (see the supplementary materials at http://www.rcjournal.com). The majority of children $(>80 \%)$ with OSAHS underwent tonsillectomies and adenoidectomies.

Children with a disease duration of $<5$ y were significantly younger than those with a disease duration of $\geq 5 \mathrm{y}$ $(P<.001)$ (Table 1). Thus, linear regression analyses were performed, which showed that age also significantly influenced disease duration. Children with a disease duration of $<5$ y had significantly higher IGF-1 and IGFBP-3 concentrations and significantly lower weights and heights 


\section{Growth and Development of Children With OSAHS}

Table 2. Comparison of Variables by Disease Duration and Time After Surgery in Children With Obstructive Sleep Apnea-Hypopnea Syndrome

\begin{tabular}{|c|c|c|c|c|c|c|}
\hline \multirow{2}{*}{$\begin{array}{l}\text { Variable and Disease } \\
\text { Duration }(\mathrm{y})\end{array}$} & \multicolumn{3}{|c|}{ Time After Surgery } & \multirow{2}{*}{ Time Effect $P$} & \multirow{2}{*}{ Group Effect $P$} & \multirow{2}{*}{$\begin{array}{c}\text { Group } \times \text { Time } \\
\text { Effect } P\end{array}$} \\
\hline & 0 Months & 3 Months & 6 Months & & & \\
\hline \multicolumn{7}{|l|}{ AHI } \\
\hline$<5$ & $16.92 \pm 11.42$ & $4.98 \pm 2.44^{*}$ & $4.03 \pm 1.30 *$ & $<.001$ & .79 & .83 \\
\hline$\geq 5$ & $17.98 \pm 9.06$ & $4.85 \pm 2.00^{*}$ & $4.05 \pm 1.59 *$ & & & \\
\hline \multicolumn{7}{|l|}{ Lowest oxygen saturation } \\
\hline$<5$ & $0.77 \pm 0.09$ & $0.86 \pm 0.05^{*}$ & $0.89 \pm 0.05^{* \dagger}$ & $<.001$ & .26 & .25 \\
\hline$\geq 5$ & $0.77 \pm 0.07$ & $0.84 \pm 0.05^{*}$ & $0.86 \pm 0.04 *$ & & & \\
\hline \multicolumn{7}{|l|}{ BMI Z score } \\
\hline$<5$ & $0.71 \pm 1.3$ & $0.67 \pm 1.26^{*}$ & $0.63 \pm 1.24 * \dagger$ & $<.001$ & .31 & .28 \\
\hline$\geq 5$ & $0.32 \pm 1.45$ & $0.27 \pm 1.46^{*}$ & $0.22 \pm 1.49^{* \dagger}$ & & & \\
\hline \multicolumn{7}{|l|}{ Weight, kg } \\
\hline$<5$ & $22.06 \pm 4.32$ & $23.02 \pm 3.82^{*}$ & $24.6 \pm 3.33 * \dagger$ & $<.001$ & $<.001$ & .06 \\
\hline$\geq 5$ & $34.23 \pm 12.16 \ddagger$ & $34.69 \pm 11.39 \ddagger$ & $35.76 \pm 10.72 * \dagger+$ & & & \\
\hline \multicolumn{7}{|l|}{ Height, cm } \\
\hline$<5$ & $113.84 \pm 7.47$ & $116.55 \pm 7.41 *$ & $120.76 \pm 8.11^{* \dagger}$ & $<.001$ & $<.001$ & .02 \\
\hline$\geq 5$ & $133.09 \pm 11.19 \ddagger$ & $134.5 \pm 10.61^{* \ddagger}$ & $137.18 \pm 11.96 * \dagger+$ & & & \\
\hline \multicolumn{7}{|l|}{ Lowest oxygen level, \% } \\
\hline$<5$ & $77.48 \pm 9.19$ & $85.44 \pm 5.1^{*}$ & $89.32 \pm 4.64 *$ & $<.001$ & .26 & .25 \\
\hline$\geq 5$ & $77.31 \pm 7.23$ & $84.12 \pm 4.8^{*}$ & $86.15 \pm 4.34 * \dagger$ & & & \\
\hline \multicolumn{7}{|l|}{$\mathrm{S}_{\mathrm{pO}_{2}}, \%$} \\
\hline$<5$ & $83.16 \pm 4.91$ & $90.04 \pm 3.36^{*}$ & $91.03 \pm 4.96^{*}$ & $<.001$ & .86 & .65 \\
\hline$\geq 5$ & $83.68 \pm 4.42$ & $89.58 \pm 3.74^{*}$ & $91.47 \pm 3.55^{* \dagger}$ & & & \\
\hline \multicolumn{7}{|l|}{ Time spent at $\mathrm{S}_{\mathrm{pO}_{2}}<90 \%$} \\
\hline$<5$ & $76.68 \pm 22.03$ & $28.15 \pm 27.22^{*}$ & $6.55 \pm 13.18^{* \dagger}$ & $<.001$ & .99 & .47 \\
\hline$\geq 5$ & $78.8 \pm 18.58$ & $23.49 \pm 22.14^{*}$ & $9.13 \pm 10.5^{* \dagger}$ & & & \\
\hline \multicolumn{7}{|l|}{ IGF-1, $\mu \mathrm{g} / \mathrm{L}$} \\
\hline$<5$ & $125.20 \pm 42.38$ & $196.92 \pm 71.46^{*}$ & $388.12 \pm 111.85^{* \dagger}$ & $<.001$ & $<.001$ & $<.001$ \\
\hline$\geq 5$ & $61.92 \pm 19.07 \ddagger$ & $88.35 \pm 27.77^{*} \ddagger$ & $150.65 \pm 52.64 * \dagger \ddagger$ & & & \\
\hline \multicolumn{7}{|l|}{ IGFBP-3, mg/L } \\
\hline$<5$ & $2.00 \pm 0.54$ & $2.75 \pm 0.65^{*}$ & $4.74 \pm 1.08 * \dagger$ & $<.001$ & $<.001$ & $<.001$ \\
\hline$\geq 5$ & $1.20 \pm 0.37 \ddagger$ & $1.71 \pm 0.53^{*}+$ & $2.91 \pm 0.88^{*}+\neq$ & & & \\
\hline $\begin{array}{l}\text { Data are presented as mean } \pm \mathrm{SD} \\
\text { * Significant difference compared } \\
\dagger \text { Significant difference compared } \\
¥ \text { Significant time point differenc } \\
\text { AHI = apnea-hypopnea index } \\
\text { BMI = body mass index } \\
\text { IGF-1 = insulin-like growth facto } \\
\text { IGFBP-3 = insulin-like growth fa }\end{array}$ & $\begin{array}{l}0 \text { months }(P<.05) \text {. } \\
3 \text { months }(P<.05) \text {. } \\
\text { ared with disease duratio }\end{array}$ & of $<5 \mathrm{y}(P<.05)$. & & & & \\
\hline
\end{tabular}

compared with children with a disease duration of $\geq 5 \mathrm{y}$ (all $P<.001)$.

\section{IGF-1, IGFBP-3, AHI, Lowest Oxygen Saturation, $\mathrm{BMI} \mathrm{Z}$ Score, and $\mathrm{S}_{\mathrm{pO}_{2}}$ Factors}

There were significant effects of time, group, and group $\times$ time on IGF-1 and IGFBP-3 concentrations (all $P<$.001) (Table 2). Regardless of disease duration, children with OSAHS had significantly higher IGF-1 and IGFBP-3 concentrations at 3 and 6 months compared with 0 , as well as at 6 months compared with 3 months (all $P<.001)$. Children with a disease duration of $<5$ y had significantly higher concentrations of IGF-1 and IGFBP-3 at each time point compared with children with a disease duration of $\geq 5$ y (all $P<.001)$.

There were significant effects of time on AHI and lowest oxygen saturation (all $P<.001$ ). Regardless of disease duration, children with OSAHS had significantly lower AHI and significantly higher lowest oxygen saturation at 3 and 6 months compared with 0 months (all $P<.001$ ). Children with a disease duration of $<5$ y also had a significantly higher lowest oxygen saturation at 6 months compared with 3 months $(P=.05)$.

There were significant effects of time on BMI Z scores (all $P<.001$ ). Regardless of disease duration, children 


\section{Growth And Development of ChILdRen With OSAHS}

with OSAHS had significantly lower BMI Z scores at 3 and 6 months compared with 0 months (all $P<.001$ ) and had significantly lower BMI Z scores at 6 months compared with 3 months (all $P<.001$ ).

There were significant effects of time and group on weight. Children with a disease duration of $<5 \mathrm{y}$ had significantly higher weights at 3 and 6 months compared with 0 months (all $P \leq .002$ ) and had significantly higher weights at 6 months compared with 3 months $(P<.001)$. Children with a disease duration of $\geq 5 \mathrm{y}$ had significantly higher weights at 6 months compared with 0 and 3 months (all $P<.001$ ). There were significant effects of time, group, and group $\times$ time on height (all $P \leq .02$ ).

Regardless of disease duration, children with OSAHS had significantly higher heights at 3 and 6 months compared with 0 months (all $P \leq .044$ ) and had significantly higher heights at 6 months compared with 3 months (all $P<.001$ ). Children with a disease duration of $<5 \mathrm{y}$ had significantly lower weights and heights at each time point compared with children with a disease duration of $\geq 5 \mathrm{y}$ (all $P<.001$ ).

There were significant effects of time on $\mathrm{S}_{\mathrm{pO}_{2}}$ factors (all $P<.001$ ). Regardless of disease duration, children with OSAHS had significantly higher percentages of lowest $\mathrm{S}_{\mathrm{pO}_{2}}$ and significantly lower percentages of time spent at $\mathrm{S}_{\mathrm{pO}_{2}}<90 \%$ at 3 and 6 months compared with 0 months $(P<.001)$. Children with a disease duration of $\geq 5 \mathrm{y}$ had significantly higher percentages of lowest $\mathrm{S}_{\mathrm{pO}_{2}}$ and lower percentages of time spent at $\mathrm{S}_{\mathrm{pO}_{2}}<90 \%$ at 6 months compared with 3 months $(P \leq .02)$. Children with a disease duration of $<5$ y also had a significantly lower percentage of time spent at $\mathrm{S}_{\mathrm{pO}_{2}}<90 \%$ at 6 months compared with 3 months $(P=.003)$.

There were significant effects of time on IGF-1 and IGFBP-3 concentrations (all $P<.001$ ) (Table 3 ). Regardless of disease severity, children with OSAHS had significantly higher IGF-1 and IGFBP-3 concentrations at 6 months compared with 0 months (all $P<.05$ ). Children with mild or moderate disease also had significantly higher IGF-1 and IGFBP-3 concentrations at 3 months compared with 0 months, whereas children with severe disease had significantly higher IGFBP-3 concentrations at 3 months compared with 0 months (all $P<.05$ ).

There were significant effects of time, group, and group $\times$ time on AHI and lowest oxygen saturation (all $P<.001$ ). Regardless of disease severity, children with OSAHS had significantly lower AHI at 3 and 6 months compared with 0 months (all $P \leq .001$ ). At 0 months, children with moderate or severe disease had significantly higher AHI compared with children with mild disease, and children with severe disease had significantly higher AHI compared with children with moderate disease (all $P<.001)$. Regardless of disease severity, children with OSAHS had significantly higher lowest oxygen saturation at 6 months compared with 0 and 3 months (all $P<.05$ ). Children with moderate and severe disease also had significantly higher lowest oxygen saturation at 3 months compared with 0 months (both $P<.001$ ). At all time points, children with severe disease had significantly lower lowest oxygen saturation compared with children with mild disease $(P<.01)$. At 0 and 6 months, children with severe disease also had significantly lower lowest oxygen saturation compared with children with moderate disease $(P<.05)$. At 0 months, children with moderate disease had significantly lower lowest oxygen saturation compared with children with mild disease $(P<.001)$.

There were significant effects of time on BMI Z scores (all $P<.001$ ). Regardless of disease severity, children with OSAHS had significantly lower BMI Z scores at 6 months compared with 0 and 3 months (all $P \leq .006$ ) and also had significantly lower BMI Z scores at 3 months compared with 0 months (all $P<.001$ ). For weight and height, there were significant effects of time (all $P<.001$ ). Regardless of disease severity, children with OSAHS had significantly higher weight at 6 months compared with 0 and 3 months (all $P \leq .02$ ); children with moderate disease had significantly higher weights at 3 months compared with 0 months $(P=.002)$. Regardless of disease severity, children with OSAHS had significantly higher heights at 6 months compared with 0 and 3 months (all $P \leq .03$ ); children with moderate and severe disease had significantly higher heights at 3 months compared with 0 months (all $P=.001$ ).

There were significant effects of time, group, and group $\times$ time on $\mathrm{S}_{\mathrm{pO}_{2}}$ factors (all $P<.046$ ). At all time points, children with severe disease had significantly lower percentages of the lowest oxygen level compared with children with mild disease $(P<.007)$. At 0 and 6 months, children with severe disease also had significantly lower percentages of the lowest oxygen level compared with children with moderate disease $(P<.05)$. At all time points, children with moderate and severe disease had significantly lower percentages of $\mathrm{S}_{\mathrm{pO}_{2}}$ compared with children with mild disease $(P<.03)$.

After making adjustments for duration, age, AHI, time spent at $\mathrm{S}_{\mathrm{pO}_{2}}<90 \%$, and BMI Z score, IGF-1 concentrations were significantly decreased at 0 and 3 months after surgery compared with those at 6 months after surgery. After adjusting for time after surgery, age, AHI, time spent at $\mathrm{S}_{\mathrm{pO}_{2}}<90 \%$, and BMI Z score, IGF-1 concentrations significantly increased in subjects with a disease duration of $<5 \mathrm{y}$ compared with those with a duration of $\geq 5 \mathrm{y}$. After adjusting for time after surgery, disease duration, AHI, time spent at $\mathrm{S}_{\mathrm{pO}_{2}}<90 \%$ and BMI Z score, IGF-1 concentrations significantly decreased with every year of age (Table 4).

After making adjustments for duration, age, AHI, time spent at $\mathrm{S}_{\mathrm{pO}_{2}}<90 \%$, and BMI Z score, IGFBP-3 concen- 


\section{Growth and Development of Children With OSAHS}

Table 3. Comparison of Variables by Disease Severity and Time After Surgery in Children With Obstructive Sleep Apnea-Hypopnea Syndrome

\begin{tabular}{|c|c|c|c|c|c|c|}
\hline \multirow{2}{*}{ Variable and Disease Severity } & \multicolumn{3}{|c|}{ Time After Surgery } & \multirow{2}{*}{ Time Effect $P$} & \multirow{2}{*}{ Group Effect $P$} & \multirow{2}{*}{$\begin{array}{l}\text { Group } \times \text { Time } \\
\text { Effect } P\end{array}$} \\
\hline & 0 Months & 3 Months & 6 Months & & & \\
\hline \multicolumn{7}{|l|}{ AHI } \\
\hline Mild & $8.46 \pm 1.13$ & $4.15 \pm 2.31^{*}$ & $3.12 \pm 0.96^{*}$ & $<.001$ & $<.001$ & $<.001$ \\
\hline Moderate & $16.02 \pm 2.58 \dagger$ & $4.61 \pm 0.9 *$ & $4.02 \pm 1.00^{*}$ & & & \\
\hline Severe & $28.53 \pm 10.39 \dagger \dagger$ & $6.08 \pm 2.72 *$ & $5.05 \pm 1.66^{*}$ & & & \\
\hline \multicolumn{7}{|l|}{ Lowest oxygen saturation } \\
\hline Mild & $0.85 \pm 0.02$ & $0.87 \pm 0.04$ & $0.9 \pm 0.04 * \S$ & $<.001$ & $<.001$ & $<.001$ \\
\hline Moderate & $0.77 \pm 0.05 \dagger$ & $0.85 \pm 0.04^{*}$ & $0.88 \pm 0.04 * \S$ & & & \\
\hline Severe & $0.7 \pm 0.08 \dagger+$ & $0.82 \pm 0.06^{* \dagger}$ & $0.85 \pm 0.05^{*} \dagger \ddagger \S$ & & & \\
\hline \multicolumn{7}{|l|}{ BMI Z score } \\
\hline Mild & $0.5 \pm 1.15$ & $0.45 \pm 1.15^{*}$ & $0.4 \pm 1.16^{*} \S$ & $<.001$ & .90 & .70 \\
\hline Moderate & $0.62 \pm 1.52$ & $0.57 \pm 1.5^{*}$ & $0.53 \pm 1.49 * \S$ & & & \\
\hline Severe & $0.4 \pm 1.51$ & $0.36 \pm 1.51 *$ & $0.32 \pm 1.51 * \S$ & & & \\
\hline \multicolumn{7}{|l|}{ Weight, kg } \\
\hline Mild & $28.97 \pm 10.6$ & $29.65 \pm 10.19$ & $31.16 \pm 9.44 * \S$ & $<.001$ & .81 & .94 \\
\hline Moderate & $26.92 \pm 10.56$ & $27.77 \pm 10.06^{*}$ & $28.97 \pm 9.85^{*} \S$ & & & \\
\hline Severe & $29.03 \pm 12.36$ & $29.61 \pm 11.27$ & $30.84 \pm 10.33 * \S$ & & & \\
\hline \multicolumn{7}{|l|}{ Height, cm } \\
\hline Mild & $126.85 \pm 12.22$ & $128.61 \pm 11.6$ & $132.34 \pm 11.51 * \S$ & $<.001$ & .43 & .99 \\
\hline Moderate & $120.95 \pm 13.08$ & $123.28 \pm 13.57^{*}$ & $126.36 \pm 14.45^{*} \S$ & & & \\
\hline Severe & $123.3 \pm 15.46$ & $125.34 \pm 13.44^{*}$ & $128.83 \pm 13.18 * \S$ & & & \\
\hline \multicolumn{7}{|l|}{ Lowest oxygen level, \% } \\
\hline Mild & $84.76 \pm 2.28$ & $86.82 \pm 3.99$ & $89.71 \pm 4.37 * \S$ & $<.001$ & $<.001$ & $<.001$ \\
\hline Moderate & $77.22 \pm 5.08 \dagger$ & $85.17 \pm 3.91^{*}$ & $88.28 \pm 4.06 * \S$ & & & \\
\hline Severe & $69.75 \pm 7.97 \dagger t$ & $82.13 \pm 5.9^{* \dagger}$ & $84.94 \pm 4.73 * \dagger+\S$ & & & \\
\hline \multicolumn{7}{|l|}{$\mathrm{S}_{\mathrm{pO}_{2}}, \%$} \\
\hline Mild & $87.77 \pm 1.39$ & $92.51 \pm 2.52^{*}$ & $93.18 \pm 3.96^{*}$ & $<.001$ & $<.001$ & .006 \\
\hline Moderate & $82.99 \pm 3.82 \dagger$ & $89.24 \pm 2.25^{* \dagger}$ & $90.63 \pm 4.87 * \dagger$ & & & \\
\hline Severe & $79.31 \pm 3.62 \dagger \ddagger$ & $87.57 \pm 3.89^{* \dagger}$ & $89.91 \pm 3.22 * \dagger \S$ & & & \\
\hline \multicolumn{7}{|l|}{ Time spent at $\mathrm{S}_{\mathrm{pO}_{2}}<90 \%, \%$} \\
\hline Mild & $61.18 \pm 26.09$ & $21.08 \pm 23.93 *$ & $4.09 \pm 6.76^{*} \S$ & $<.001$ & $<.001$ & .05 \\
\hline Moderate & $81.73 \pm 9.37 \dagger$ & $22.91 \pm 18.63^{*}$ & $5.63 \pm 9.14 * \S$ & & & \\
\hline Severe & $90.91 \pm 3.91 \dagger$ & $33.99 \pm 30.16^{* \dagger}$ & $14.39 \pm 16^{*} \S$ & & & \\
\hline \multicolumn{7}{|l|}{ IGF-1, $\mu \mathrm{g} / \mathrm{L}$} \\
\hline Mild & $93.82 \pm 37.52$ & $150.12 \pm 81.44^{*}$ & $281.88 \pm 147.47 * \S$ & $<.001$ & .14 & .21 \\
\hline Moderate & $109.06 \pm 58.42$ & $158.56 \pm 83.39^{*}$ & $303.89 \pm 158.79 * \S$ & & & \\
\hline Severe & $73.88 \pm 28.51$ & $113.38 \pm 56.91$ & $209.88 \pm 124.14 * \S$ & & & \\
\hline \multicolumn{7}{|l|}{ IGFBP-3, mg/L } \\
\hline Mild & $1.76 \pm 0.57$ & $2.48 \pm 0.78^{*}$ & $4.28 \pm 1.52 * \S$ & $<.001$ & .10 & .32 \\
\hline Moderate & $1.67 \pm 0.72$ & $2.27 \pm 0.87^{*}$ & $3.74 \pm 1.24 * \S$ & & & \\
\hline Severe & $1.33 \pm 0.45$ & $1.89 \pm 0.6^{*}$ & $3.38 \pm 1.16 * \S$ & & & \\
\hline $\begin{array}{l}\text { Data are presented as mean } \pm \text { SD. } \\
\text { * Significant difference compared with } 0 \\
\dagger \text { Significant time point difference compa } \\
\text { † Significant time point difference compa } \\
\S \text { Significant difference compared with } 3 \\
\text { AHI = apnea-hypopnea index } \\
\text { BMI = body mass index } \\
\text { IGF-1 = insulin-like growth factor- } 1 \\
\text { IGFBP-3 = insulin-like growth factor-bin }\end{array}$ & $\begin{array}{l}\text { onths }(P<.05) \text {. } \\
\text { d with mild disease severit } \\
\text { d with moderate disease se } \\
\text { onths }(P<.05) \text {. } \\
\text { ng protein-3 }\end{array}$ & $\begin{array}{l}\text { of }<5 \mathrm{y}(P<.05) \\
\text { erity of }<5 \mathrm{y}(P<.05) \text {. }\end{array}$ & & & & \\
\hline
\end{tabular}

trations significantly decreased with time after surgery at 0 and 3 months after surgery compared with 6 months after surgery. After making adjustments for time after surgery, disease duration, $\mathrm{AHI}$, time spent at $\mathrm{S}_{\mathrm{pO}_{2}}<90 \%$, and $\mathrm{BMI}$ $\mathrm{Z}$ score, IGFBP-3 concentrations significantly decreased with every year of age. After adjusting for time after sur- 
Table 4. Results of Multivariate Linear Mixed Model

\begin{tabular}{|c|c|c|}
\hline & $\beta$ (SE) & $P$ \\
\hline \multicolumn{3}{|l|}{ IGF-1 } \\
\hline \multicolumn{3}{|l|}{ Time after surgery } \\
\hline 0 months (reference of 6 months) & $-197.78(38.23)$ & $<.001$ \\
\hline 3 months (reference of 6 months) & $-162.89(20.58)$ & $<.001$ \\
\hline Disease duration of $<5 \mathrm{y}$ (reference of $\geq 5 \mathrm{y}$ ) & $79.13(37)$ & .04 \\
\hline Age & $-28.00(8.89)$ & .003 \\
\hline AHI & $0.06(1.52)$ & .97 \\
\hline Time spent at $\mathrm{S}_{\mathrm{pO}_{2}}<90 \%$ & $-52.97(47.67)$ & .27 \\
\hline BMI Z score & $-6.47(7.85)$ & .41 \\
\hline \multicolumn{3}{|l|}{ IGFBP-3 } \\
\hline \multicolumn{3}{|l|}{ Time after surgery } \\
\hline 0 months (reference of 6 months) & $-2.49(0.36)$ & $<.001$ \\
\hline 3 months (reference of 6 months) & $-1.89(0.18)$ & $<.001$ \\
\hline Disease duration of $<5$ y (reference of $\geq 5 \mathrm{y}$ ) & $0.42(0.43)$ & .33 \\
\hline Age & $-0.29(0.1)$ & .007 \\
\hline AHI & $0.001(0.01)$ & .007 \\
\hline Time spent at $\mathrm{S}_{\mathrm{pO}_{2}}<90 \%$ & $-0.7(0.45)$ & .94 \\
\hline BMI Z score & $0.08(0.09)$ & .12 \\
\hline \multicolumn{3}{|l|}{$\begin{array}{l}\text { IGF-1 = insulin-like growth factor-1 } \\
\text { AHI = apnea-hypopnea index } \\
\text { BMI = body mass index } \\
\text { IGFBP-3 = insulin-like growth factor-binding protein-3 }\end{array}$} \\
\hline
\end{tabular}

gery, disease duration, age, time spent at $\mathrm{S}_{\mathrm{pO}_{2}}<90 \%$, and BMI Z score, IGFBP-3 concentrations significantly increased with increases in AHI (see Table 4).

\section{Discussion}

In this study, we examined IGF-1 and IGFBP-3 concentrations in children with OSAHS before and after surgical intervention. We then compared the concentrations of these mediators of growth with regard to OSAHS disease duration and disease severity. To the best of our knowledge, this is the first study to investigate the effect of OSAHS duration on growth. After surgery (bilateral tonsillectomy and adenoidectomy or adenoidectomy), the concentrations of both IGF-1 and IGFBP-3 increased. Our results also indicate that age and disease duration (but not disease severity) may affect IGF-1 and IGFBP-3 concentrations in children with OSAHS before and after surgery.

Our findings that IGF-1 and IGFBP-3 concentrations increased in children with OSAHS after adenoidectomy and/or tonsillectomy are consistent with findings from several other studies. For instance, Gümüssoy et a ${ }^{15}$ reported that serum levels of IGF-1 and IGFBP-3 in children with sleep-disordered breathing were significantly increased at 6 months after adenoidectomy and tonsillectomy. Likewise, Nieminen et al ${ }^{12}$ found that children with symptoms of OSAHS who underwent adenoidectomy and tonsillectomy had significantly higher IGF-1 and IGFBP-3 con- centrations at 6 months after surgery compared with children who did not undergo surgery. Bar et al ${ }^{16}$ also reported that both IGF-1 concentrations and body weight were significantly increased in children with OSAHS who underwent adenoidectomy and tonsillectomy. These changes in IGF-1 and IGFBP-3 and the significant improvements in AHI and lowest oxygen saturation (indicating relief of airway obstruction) emphasize the importance of surgical intervention for the treatment of OSAHS in children.

We found that the mean serum concentrations of IGF-I and IGFBP-3 significantly increased with time after surgery (particularly between 3 and 6 months) and were significantly lower among children with a disease duration of $\geq 5 \mathrm{y}$ at each assessment point. These findings suggest that children who have had OSAHS for a longer period of time experience more pronounced perturbations in underlying mediators of growth. Furthermore, recovery of IGF-1 and IGFBP-3 concentrations after surgery is clearly much slower in these children. Indeed, further study is needed to determine how long it takes for IGF-1 and IGFBP-3 concentrations to recover fully in children with a disease duration of $\geq 5 \mathrm{y}$. Taken together, our findings regarding disease duration lead us to suggest that prompt surgical intervention may be of significant benefit to normalize IGF-1 and IGFBP-3 concentrations (and thus help maintain normal growth) in children with OSAHS caused by adenoidal and/or tonsillar hypertrophy. Further studies should be conducted to determine whether delaying sur- 


\section{Growth and Development of Children With OSAHS}

gical intervention could potentially impact the growth and development of children or further aggravate the problems of development in children with growth retardation.

Before surgery, we found that serum IGF-I and IGFBP-3 concentrations were near the lower limit of normal in children with OSAHS. Of note, however, there were no significant differences in IGF-1 and IGFBP-3 concentrations regarding disease severity as determined by polysomnography. Furthermore, after surgery, IGF-1 and IGFBP-3 concentrations increased over time to a similar extent, regardless of disease severity. These findings suggest that the severity of OSAHS, at least as determined by polysomnography, is not a major determinant of disease impact on the important mediators of growth and development: IGF-1 and IGFBP-3. In this context, surgery would appear to be just as likely to benefit children with mild disease as children with severe disease.

Bedogni et al ${ }^{17}$ found that IGF-1 concentrations rose with increasing age in their cross-sectional study of 24,403 subjects $0-18$ y old. Conversely, IGF- 1 and IGFBP- 3 concentrations decreased following surgery in our study as the children aged (after making adjustments for time after surgery, disease duration, $\mathrm{AHI}$, time spent at $\mathrm{S}_{\mathrm{pO}_{2}}<90 \%$, and BMI $\mathrm{Z}$ score). The findings of Blum et $\mathrm{al}^{11}$ may explain this phenomenon. They hypothesized that there may be increased sensitivity of IGF-I regulation by growth hormone for individuals who have reached puberty compared with their prepubertal counterparts.

OSAHS is a progressive disease, and its severity can change over time theoretically, but monitoring the entire duration of the patient's disease is currently not possible. We can only determine children's OSAHS severity at the time of study visits. Children who had not entered puberty have different IGF-1 and IGFBP-3 levels at different ages, so different disease severities were compared horizontally among the 3 groups. The age distribution of each group must be balanced, so we conducted a statistical analysis to minimize the impact of the age factor. In theory, when 2 groups with different disease durations are compared, the impact of age factor can affect outcomes. Theoretically, the greater the age, the higher the levels of IGF-1 and IGFBP-3. However, the results we obtained from the statistical analyses showed that IGF-1 and IGFBP-3 levels in older children (with longer disease durations) are lower than those in younger children (with shorter disease durations). These data suggest that even with the age factors, there are still significant differences between the 2 groups.

In clinical diagnosis, the first consideration of physicians is often the impact of the severity of OSAHS on complications. In the case of patients with the same disease duration, we assume that complications are usually more severe in patients with severe OSAHS compared with those in patients with mild disease. However, due to complications of OSAHS such as chronic injuries, even in patients with severe OSAHS of shorter durations, the damage will not be necessarily greater than that for patients with mild OSAHS of longer durations. Therefore, if one considers the severity of the disease as an independent factor, the results obtained above are also reasonable. As we can see from the above analysis, determining the extent of damage caused by complications of OSAHS by judging one single factor is prone to subjective bias. Therefore, disease duration should be taken into account to make a more objective judgment.

IGF-1 and IGFBP-3 levels increased after intervention, whereas BMI-Z scores decreased. Another study reported a high correlation between age-adjusted and sex-adjusted SD scores for IGF-I and leptin in obese children (modulated by sex, pubertal stage, and body weight). ${ }^{10}$ Another study found that weight and height gains exceeded expectations in $75 \%$ of subjects, especially during the first year following tonsillectomy. ${ }^{4} \mathrm{~A}$ review of 20 studies found that standardized height and weight and IGF-1 and IGFBP-3 concentrations increased significantly after adenotonsillectomy. ${ }^{7}$ Because BMI actually decreased in our study, this suggests that surgical intervention had varying effects on height and weight.

Our results concerning AHI and lowest oxygen saturation are unsurprising in that these variables improved regardless of disease duration or severity after surgery and that $\mathrm{AHI}$ and lowest oxygen saturation at baseline differed by disease severity. The improvement in AHI after surgery was evident at 3 months; there was little change between 3 and 6 months. Interestingly, the recovery of lowest oxygen saturation did seem to be affected by both time and disease severity, with measures increasing with time after surgery and generally being lower in children with severe disease compared with those with mild disease.

This study has several limitations that warrant mention. Although our analyses were appropriately powered, they included a relatively small number of children. Further larger scale studies are needed to confirm and extend the findings presented herein. We chose to use the normal reference ranges of IGF-1 and IGFBP-3 provided by the manufacturer of the detection device. The length of follow-up was limited to 6 months after surgery. Longer term changes (ie, $>6$ months) in IGF-1 and IGFBP-3 should be assessed, in addition to direct measures of growth and development. Children with a disease duration of $<5 y$ were significantly younger than those with a disease duration of $\geq 5 \mathrm{y}$. This age difference may have confounded our analyses regarding disease duration. The subject cohort was composed of children who experienced OSAHS onset at $3 \mathrm{y}$ of age. Therefore, the data cannot necessarily be extrapolated to children who experienced OSAHS with earlier or later onsets. A final limitation is the retrospective study design (retrospective analysis of prospective data). Future prospective studies on this topic are needed to ob- 


\section{Growth and Development of Children With OSAHS}

viate the inherent biases and limitations associated with retrospective studies.

\section{Conclusions}

In summary, OSAHS caused by adenoidal and/or tonsillar hypertrophy significantly reduces concentrations of 2 key mediators of growth and development in children: IGF-1 and IGFBP-3. Adenoidectomy and/or tonsillectomy significantly increased the concentrations of IGF-1 and IGFBP-3, regardless of disease severity. Disease duration was an important factor in the level of increase observed in these parameters. Our findings suggest that OSAHSrelated perturbations in IGF-1 and IGFBP-3 are more pronounced and slower to recover in children with a longer disease duration. Prompt treatment may help alleviate the potential impact of the aforementioned changes on growth and development.

\section{REFERENCES}

1. Brunetti L, Rana S, Lospalluti ML, Pietrafesa A, Francavilla R, Fanelli M, Armenio L. Prevalence of obstructive sleep apnea syndrome in a cohort of 1,207 children of southern Italy. Chest 2001; 120(6):1930-1935.

2. Anuntaseree W, Rookkapan K, Kuasirikul S, Thongsuksai P. Snoring and obstructive sleep apnea in Thai school-age children: prevalence and predisposing factors. Pediatr Pulmonol 2001;32(3):222-227.

3. Greenfeld M, Tauman R, DeRowe A, Sivan Y. Obstructive sleep apnea syndrome due to adenotonsillar hypertrophy in infants. Int J Pediatr Otorhinolaryngol 2003;67(10):1055-1060.

4. Ahlqvist-Rastad J, Hultcrantz E, Melander H, Svanholm H. Body growth in relation to tonsillar enlargement and tonsillectomy. Int J Pediatr Otorhinolaryngol 1992;24(1):55-61.

5. Alexander NS, Schroeder JW Jr. Pediatric obstructive sleep apnea syndrome. Pediatr Clin North Am 2013;60(4):827-840.

6. Tan HL, Gozal D, Kheirandish-Gozal L. Obstructive sleep apnea in children: a critical update. Nat Sci Sleep 2013;5:109-123.
7. Bonuck KA, Freeman K, Henderson J. Growth and growth biomarker changes after adenotonsillectomy: systematic review and meta-analysis. Arch Dis Child 2009;94(2):83-91.

8. Barkan AL, Beitins IZ, Kelch RP. Plasma insulin-like growth factorI/somatomedin-C in acromegaly: correlation with the degree of growth hormone hypersecretion. J Clin Endocrinol Metab 1988;67(1):69-73.

9. Vontetsianos HS, Davris SE, Christopoulos GD, Dacou-Voutetakis C. Improved somatic growth following adenoidectomy and tonsillectomy in young children. Possible pathogenetic mechanisms. Hormones 2005;4(1):49-54.

10. Woelfle JF, Harz K, Roth C. Modulation of circulating IGF-I and IGFBP-3 levels by hormonal regulators of energy homeostasis in obese children. Exp Clin Endocrinol Diabetes 2007;115(1):17-23.

11. Blum WF, Albertsson-Wikland K, Rosberg S, Ranke MB. Serum levels of insulin-like growth factor I (IGF-I) and IGF binding protein 3 reflect spontaneous growth hormone secretion. J Clin Endocrinol Metab 1993;76(6):1610-1616.

12. Nieminen $P$, Löppönen $T$, Tolonen U, Lanning P, Knip M, Löppönen $\mathrm{H}$. Growth and biochemical markers of growth in children with snoring and obstructive sleep apnea. Pediatrics 2002;109(4):e55.

13. Van Cauter E, Latta F, Nedeltcheva A, Spiegel K, Leproult R, Vanderbril C, et al. Reciprocal interactions between the $\mathrm{GH}$ axis and sleep. Growth Horm IGF Res 2004;14 Suppl A:S10-S17.

14. Editorial Board of Chinese Journal of Otorhinolaryngology and Head and Neck Surgery, Chinese Otorhinolaryngology of Chinese Medical Association. [Draft guidelines for the diagnosis and treatment of pediatric sleep apnea hypopnea syndrome (Urumqi)]. Zhonghua Er Bi Yan Hou Tou Jing Wai Ke Za Zhi 2007;42(2):83-84. Article in Chinese.

15. Gümüssoy M, Atmaca S, Bilgici B, Unal R. Changes in IGF-I, IGFBP-3 and ghrelin levels after adenotonsillectomy in children with sleep disordered breathing. Int J Pediatr Otorhinolaryngol 2009; 73(12):1653-1656.

16. Bar A, Tarasiuk A, Segev Y, Phillip M, Tal A. The effect of adenotonsillectomy on serum insulin-like growth factor-I and growth in children with obstructive sleep apnea syndrome. J Pediatr 1999; 135(1):76-80.

17. Bedogni G, Giannone G, Maghnie M, Giacomozzi C, Di Iorgi N, Pedicelli S, et al. Serum insulin-like growth factor-I (IGF-I) reference ranges for chemiluminescence assay in childhood and adolescence. Data from a population of in- and out-patients. Growth Horm IGF Res 2012;22(3-4):134-138. 